\title{
Acute transverse myelitis and subacute thyroiditis associated with dengue viral infection: A case report and literature review
}

\author{
ZHIMING MO, YAXIAN DONG, XIAOLIAN CHEN, HUIYAN YAO and BIN ZHANG \\ Department of Neurology, The Fourth Affiliated Hospital of Guangzhou Medical University, \\ Guangzhou, Guangdong 510150, P.R. China
}

Received May 21, 2015; Accepted July 15, 2016

DOI: $10.3892 / e t m .2016 .3604$

\begin{abstract}
Acute transverse myelitis is a rare manifestation of dengue infection. To the best of our knowledge, only 6 cases of acute transverse myelitis as a manifestation of dengue infection have been reported thus far. The present study described a case of acute transverse myelitis complicated with subacute thyroiditis 6 days after the onset of dengue viral infection. In addition, the available literature was searched to identify similar previous cases. Treatment with intravenous pulse methylprednisolone immunoglobulin plasmapheresis and physiotherapy resulted in partial recovery at 3 months post-infection. In conclusion, the involvement of dengue infection should be considered in patients who develop central nervous system manifestations during or after the recovery period of dengue infection. Furthermore, since methylprednisolone and immunoglobulin are effective during the active phase of the infection, prompt diagnosis and initiation of treatment are crucial.
\end{abstract}

\section{Introduction}

Dengue is the most common arboviral disease that is caused by dengue virus (1), and occurs in Southeast Asia, East and West Africa, the Caribbean and the Americas (2). Dengue fever and dengue hemorrhagic fever are increasingly recognized diseases in Asia (2). Various neurologic complications of dengue viral infection have been reported, including Guillain-Barré syndrome (GBS), encephalitis, encephalopathy, myelitis, meningitis, acute disseminated encephalomyelitis (ADEM), polyneuropathy, and facial and ulnar mononeuropathy (3-6). However, the involvement of the spinal cord and thyroid in patients infected with dengue has been rarely reported. To the best of our knowledge, only 6 cases of transverse myelitis in association with dengue infection have been previously

Correspondence to: Dr Yaxian Dong, Department of Neurology, The Fourth Affiliated Hospital of Guangzhou Medical University, 63 Asian Games Road, Guangzhou, Guangdong 510150, P.R. China E-mail: wedding2@163.com

Key words: dengue, acute transverse myelitis, subacute thyroiditis reported in the literature, one of which was complicated with subacute thyroiditis. The present study describes the case of a 65-year-old patient who developed transverse myelitis complicated with subacute thyroiditis 6 days following dengue infection. In addition, the current study reviewed the previous relevant cases in the literature in order to elucidate the pathogenesis of spinal cord involvement in dengue viral infection.

\section{Case report}

A 65-year-old male patient presented at the Fourth Affiliated Hospital of Guangzhou Medical University (Guangzhou, China) in October 2014 with acute paraplegia and sensory loss. The patient had experienced an acute onset of fever $\left(39^{\circ} \mathrm{C}\right)$ 6 days before admission; in addition, he had previously suffered from myocardial infarction, and had undergone stent implantation 4 months prior to admission. Initial blood count analysis, performed on admission, showed a normal total leukocyte count, a low platelet count $\left(98 \times 10^{9}\right.$ cells/l), and positive dengue antigen and IgM (Table I). At 4 days before admission, the fever was resolved and the patient developed acute paraplegia with sensory loss after 2 days.

Neurological evaluation at admission by manual muscle testing (7), revealed grade 4/5 flaccid paralysis of the lower limbs with an absence of knee and ankle jerk reflexes. In the 2 days after admission, the patient developed muscle strength of grade 1/5 in the bilateral lower limbs, as well as T4-level sensory deficit followed by acute urinary retention. However, examination of the upper extremities, cranial nerves, cerebellum and neck showed no remarkable finding. Weakness of lower limbs was gradually aggravated, reaching grade $0 / 5$ by 4 day of admission.

Serological testing for anti-human immunodeficiency virus antibodies with the Venereal Disease Research Laboratory test was negative. Immunological testing for anti-dengue IgM/IgG and dengue antigen in the serum at admission were positive; however, these were negative in the cerebrospinal fluid (CSF) on days 2 and 4 after admission. NMDA receptor for encephalitis and aquaporin 4 antibodies were negative in both the serum and CSF. The visual evoked potential (VEP) was also negative.

Magnetic resonance imaging (MRI) of the cervical spinal cord was performed on day 5 after admission and showed hypersignal intensity on T2 weighted images at C3-C5 (Fig. 1A). 
CSF analysis on day 2 of admission revealed a clear fluid with a pressure of $14 \mathrm{cmH}_{2} \mathrm{O}$ and a leukocyte count of $24.8 \times 10^{6}$ cells $/ 1$, including $45 \%$ mononuclear cells and $55 \%$ polynuclear cells. In addition, the CSF glucose and total protein levels were $4.49 \mathrm{mmol} / \mathrm{l}$ and $791 \mathrm{mg} / \mathrm{l}$, respectively. CSF cultures were negative for bacteria and fungi. Furthermore, serological analyses on admission revealed increased FT4, thyroglobulin antibody (TGAb) and thyroid microsomal antibody (TMAb) levels, as well as reduced human thyroid-stimulating hormone (hTSH), thyroglobulin (Tg) and thyroxine-binding globulin (TBG) levels. However, no antinuclear, anti-double stranded DNA and anti-ganglioside antibodies were detected, and a thyroid ultrasound test, conducted on day 5 of admission, was negative (Fig. 1B).

According to the aforementioned findings, the patient was diagnosed with acute transverse myelitis complicated with subacute thyroiditis in association with a dengue viral infection. The patient was treated with intravenous pulse methylprednisolone (1 g/day; Pfizer Manufacturing NV, Puurs, Belgium) and immunoglobulin (20 g/day; Shuanglin Biological Pharmaceutical Co., Ltd., Zhanjiang, China) for 5 consecutive days from day 5 of admission. After 1 month of treatment, an MRI scan of the cervical spinal cord demonstrated a decreased high-intensity signal (Fig. 2) compared with the value at admission. CSF analysis showed a leukocyte count of $8 \times 10^{6}$ cells/1, glucose level of $3.23 \mathrm{mmol} / \mathrm{l}$ and total protein of $386 \mathrm{mg} / \mathrm{l}$.

At 3 months after the onset of symptoms, the patient's lower limbs were graded as $1 / 5$ after receiving intensive physiotherapy. The patient presented a T12-level sensory deficit and the sphincter function was normal; however, little improvement was observed after 4 months of follow-up.

Written informed consent for this case report was obtained from the patient's family. Approval for this study was provided by the Ethics Committee of the Fourth Affiliated Hospital of Guangzhou Medical University.

\section{Discussion}

Dengue is an infectious disease caused by a flavivirus that results in a spectrum of clinical presentations from subclinical to dengue hemorrhagic fever, and subsequently dengue shock syndrome. The neurological complications of dengue include para-infectious encephalitis, GBS and ADEM; however, transverse myelitis is rarely involved. In the present study, a literature review identified 6 reported cases (8-13) of transverse myelitis attributed to dengue infection between 1996 and 2014, in addition to the present case. Table II provides an overview of the current and previous cases. The PubMed database (www.ncbi.nlm.nih.gov/pubmed) was searched for 'transverse myelitis/TM' and 'dengue', and full-text versions of all cases were carefully analyzed. Studies involving central nervous neurological disorders that did not report appropriate medical evidence were excluded. All patients in the current and 6 previous studies had recently experienced dengue infection, with 6 adult and 1 pediatric cases identified (8-13). The time lag between infection and transverse myelitis symptoms varied between 3 and 16 days (mean, 7 days). As shown in Table II, among the 6 previous cases, there were 2 Singaporean patients (8,9), 1 Thai (10), 1 Malaysian Chinese (11) and 2 Japanese $(12,13)$. Cases initially considered to be dengue were admitted with various neurological symptoms, including spastic paraparesis (in 1 case) and flaccid paraparesis (6 cases). However, not all patients experienced typical dengue hemorrhagic fever and dengue shock syndrome prior to the onset of neurological symptoms. A careful analysis of the reported cases did not reveal any predisposing factor for the illness. All patients were found to have a low platelet count and dengue infection. In 4 out of 7 cases [the current case plus 3 others $(9,10,12)]$ MRI of the spinal cord was used to confirm the diagnosis, and hyperintensity in T2-weighted images supported the diagnosis of transverse myelitis. Furthermore, CSF analysis revealed signs of inflammation. Intravenously administered Ig methylprednisolone and/or plasmapheresis were the preferential treatments in 4 of the 7 cases [methylprednisolone in 2 cases $(8,10)$, plasmapheresis in 1 case $(12)$, and Ig methylprednisolone plus plasmapheresis in the present case). In total, 5 patients recovered completely $(8-11,13)$. However, the case reported in the present study showed a partial recovery at 3 months post-infection.

In the current patient, transverse myelitis was diagnosed based on the following typical symptoms: Transiently decreased platelet count, positive dengue antigen and $\mathrm{IgM} / \mathrm{IgG}$, and according to the features of an MRI scan of the spinal cord. To the best of our knowledge, the current study reported the first case suffering from transverse myelitis combined with dengue fever in mainland China.

Dengue is endemic in Southeast Asia, East and West Africa, the Caribbean and the Americas (2). However, all the cases reported in the literature involve Asian patients. Therefore, the Asian population appears to be prone to autoimmune injury of the spinal cord. Thrombocytopenia occurred in all previously reported cases, with the exception of the case described by Larik et al (9). However, normal leukocyte count was obtained in 5 cases of transverse myelitis $(8,10-12)$. The possible pathogenesis may be due to superinfection or co-infection, including urinary tract infection, blood-stream infection and pneumonia. Notably, leukocytosis was identified as a sign of severe dengue infection in a Taiwanese study (14); however, it cannot be ruled out that spinal cord injury was caused directly by dengue virus. According to the comparison of cases shown in Table II, para-infectious transverse myelitis also appears to be associated with flaccid paraparesis, while post-infectious myelitis may be associated with spastic paraparesis.

Thyroid involvement in dengue has been rarely reported. Subacute thyroiditis has been suggested to be a complication of expanded dengue syndrome (15). The present study detected increased FT4, TGAb and TMAb levels, as well as reduced hTSH, Tg and TBG levels in the patient, without any history of thyroiditis, which is as an autoimmune disease. Transverse myelitis has been shown to be associated with autoimmune reaction post-infection $(3,16)$. Furthermore, abnormal thyroid function is presumed to be caused by viral infection or by an inflammatory process, and multiple cases have suggested its association with coxsackievirus infection, mumps, measles, adenovirus and dengue viral infection (17-20).

The mechanisms of viral transmission and neuronal injury induced by dengue virus are relatively unknown. In the early stages, a direct pathogenic effect of dengue virus on neural tissues is indicated by the presence of viral antigen in the CSF (12). All cases identified by the literature review, as well as 
Table I. Main biochemical results of exams carried out in a patient with dengue and transverse myelitis.

\begin{tabular}{|c|c|c|c|c|c|c|c|c|c|c|c|}
\hline \multirow[b]{2}{*}{ Parameter } & \multicolumn{10}{|c|}{ Days after admission } & \multirow{2}{*}{$\begin{array}{l}\text { Reference } \\
\text { value }\end{array}$} \\
\hline & 0 & 1 & 2 & 3 & 4 & 5 & 6 & 7 & 12 & 39 & \\
\hline Leukocytes, x109/1 & 8.7 & 7.76 & 9.07 & 11.18 & 10.01 & 11.29 & 7.63 & 7.58 & 5.33 & 6.98 & $3.5-9.5$ \\
\hline Platelets, x $10^{9} / 1$ & 98 & 160 & 171 & 215 & 220 & 226 & 230 & 239 & 204 & 254 & $125-350$ \\
\hline Hematocrit, \% & 39.7 & 36.5 & 38.8 & 38.3 & 36.2 & 34.4 & 35.3 & 33.6 & 38.5 & 35.7 & $40-50$ \\
\hline ALT, U/1 & - & 23 & - & - & - & - & 29 & - & 28 & & $9-60$ \\
\hline$\gamma-\mathrm{GT}, \mathrm{U} / \mathrm{l}$ & - & 46 & - & - & - & - & 28 & - & 35 & & $10-60$ \\
\hline BUN, mmol/l & 4.1 & 2.5 & 3.8 & 4.9 & 5 & 5.7 & 6.4 & 6.8 & 7.1 & 3.8 & $2.86-8.2$ \\
\hline Creatinine, $\mu \mathrm{mol} / 1$ & 78 & 56 & 62 & 56 & 56 & 44 & 42.7 & 58 & 52 & 50 & $62-115$ \\
\hline Serum GLU, mmol/1 & 6.48 & 6.76 & 11.73 & 9.05 & 7.69 & 9.4 & 7.92 & 7.87 & 4.81 & 7.63 & $3.89-6.11$ \\
\hline FT3, pmol/l & 5.10 & & & & & & & & 3.65 & & $3.8-6$ \\
\hline FT4, pmol/1 & 20.13 & & & & & & & & 23.10 & & 7.9-14.4 \\
\hline hTSH, pmol/l & 0.09 & & & & & & & & 0.29 & & $0.34-5.6$ \\
\hline TGAb, $\%$ & 49.40 & & & & & & & & & & $0-30$ \\
\hline TMAb, $\%$ & 31.70 & & & & & & & & & & $0-20$ \\
\hline TPOAb, U/ml & 33.77 & & & & & & & & & & $0-34$ \\
\hline $\mathrm{Tg}, \mathrm{ng} / \mathrm{ml}$ & 1.72 & & & & & & & & & & $3.5-77$ \\
\hline TRAb, U/1 & 0.59 & & & & & & & & & & $<1.75$ \\
\hline $\mathrm{TBG}, \mu \mathrm{g} / \mathrm{ml}$ & 9.96 & & & & & & & & & & 13-39 \\
\hline DENV-Ag & $(+)$ & & & & & $(-)$ & & & $(-)$ & $(-)$ & Negative \\
\hline DENV-IgM & $(+)$ & & & $(+)$ & & & & & $(+)$ & $(+)$ & Negative \\
\hline DENV-IgG & $(-)$ & & & & & & & & $(+)$ & $(+)$ & Negative \\
\hline CSF WBC, $\times 10^{6} / 1$ & & & 24.8 & & 8 & & & & & 1 & 0-8 \\
\hline CSF TP, mg/l & & & 791 & & 303 & & & & & 386 & $150-450$ \\
\hline CSF GLU, mmol/1 & & & 4.49 & & 4.58 & & & & & 3.23 & $2.5-4.5$ \\
\hline CSF ADA, U/1 & & & 0.2 & & 0.3 & & & & & 4.2 & $0-5$ \\
\hline CSF DENV-IgM & & & $(-)$ & & $(-)$ & & & & & $(-)$ & Negative \\
\hline CSF DENV-IgG & & & $(-)$ & & $(-)$ & & & & & $(-)$ & Negative \\
\hline CSF DENV-Ag & & & $(-)$ & & $(-)$ & & & & & $(-)$ & Negative \\
\hline
\end{tabular}

ALT, alanine aminotransferase; $\gamma$-GT, $\gamma$-glutamyl transferase; BUN, blood urea nitrogen; GLU, glucose; hTSH, human thyroid-stimulating hormone; TGAb, thyroglobulin antibody; TMAb, thyroid microsomal antibody; TPOAb, thyroperoxidase antibody; Tg, thyroglobulin; TRAb, thyroid stimulating hormone receptor antibody; TBG, thyroxine-binding globulin; DENV, dengue virus; Ag, antigen; Ig, immunoglobulin; CSF, cerebrospinal fluid; WBC, white blood cell; TP, total protein; ADA, adenosine deaminase; (+), positive result; (-), negative result.
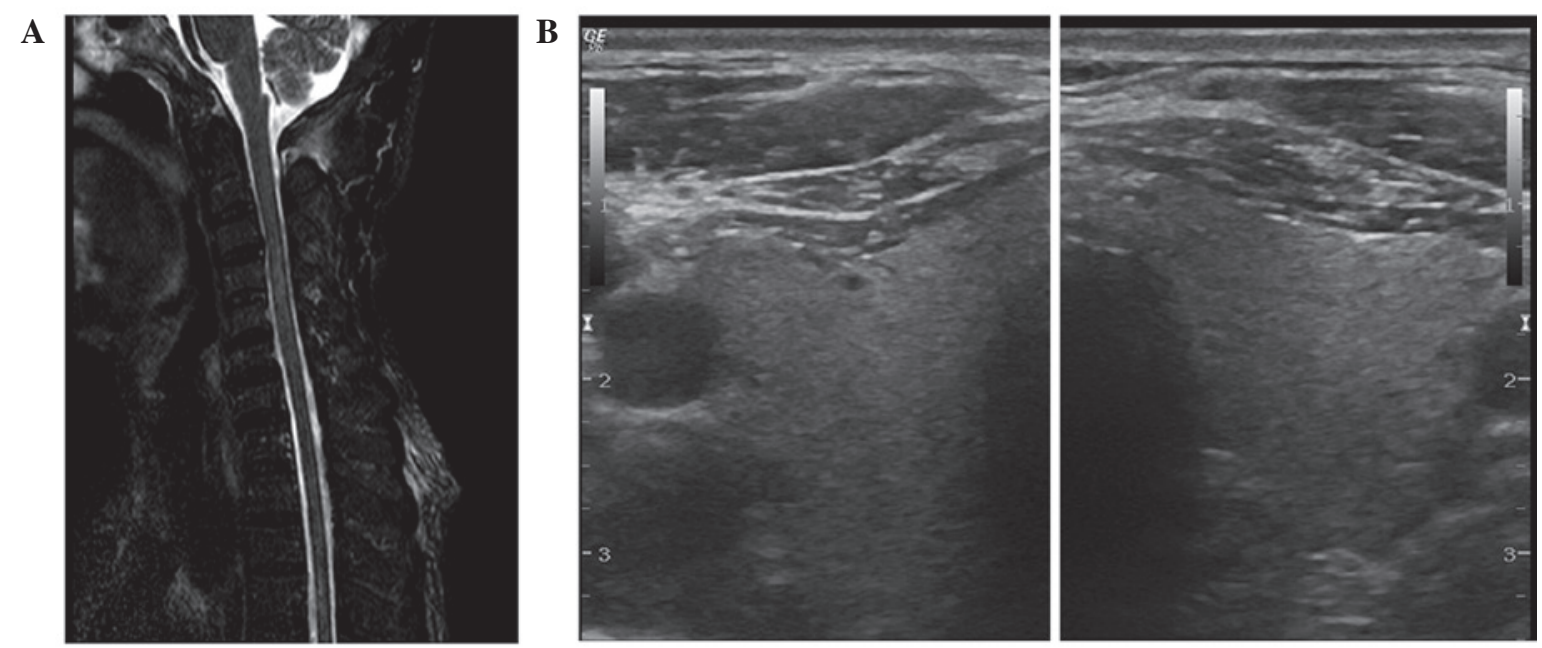

Figure 1. (A) T2-weighted magnetic resonance image showed a high-intensity signal at C3-C5 in the spinal cord and supported the diagnosis. (B) A thyroid ultrasound test demonstrated normal thyroid and uniform signal with no lesion. 

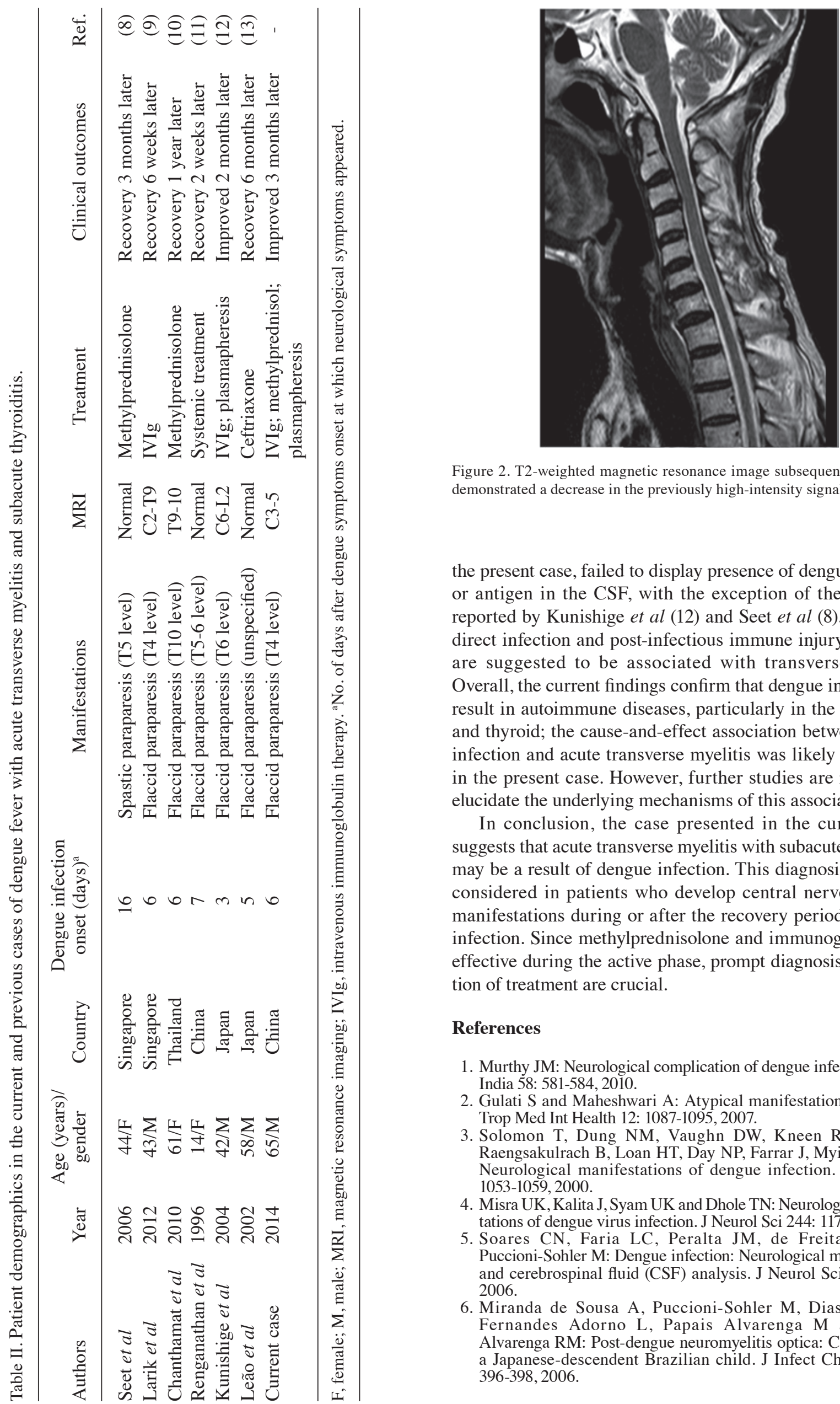

Figure 2. T2-weighted magnetic resonance image subsequent to treatment demonstrated a decrease in the previously high-intensity signal.

the present case, failed to display presence of dengue $\operatorname{IgM} / \operatorname{IgG}$ or antigen in the CSF, with the exception of the two cases reported by Kunishige et al (12) and Seet et al (8). Therefore, direct infection and post-infectious immune injury in dengue are suggested to be associated with transverse myelitis. Overall, the current findings confirm that dengue infection can result in autoimmune diseases, particularly in the spinal cord and thyroid; the cause-and-effect association between dengue infection and acute transverse myelitis was likely established in the present case. However, further studies are required to elucidate the underlying mechanisms of this association.

In conclusion, the case presented in the current study suggests that acute transverse myelitis with subacute thyroiditis may be a result of dengue infection. This diagnosis should be considered in patients who develop central nervous system manifestations during or after the recovery period of dengue infection. Since methylprednisolone and immunoglobulin are effective during the active phase, prompt diagnosis and initiation of treatment are crucial.

\section{References}

1. Murthy JM: Neurological complication of dengue infection. Neurol India 58: 581-584, 2010.

2. Gulati S and Maheshwari A: Atypical manifestations of dengue. Trop Med Int Health 12: 1087-1095, 2007.

3. Solomon T, Dung NM, Vaughn DW, Kneen R, Thao LT, Raengsakulrach B, Loan HT, Day NP, Farrar J, Myint KS, et al: Neurological manifestations of dengue infection. Lancet 355: $1053-1059,2000$

4. Misra UK, Kalita J, Syam UK and Dhole TN: Neurological manifestations of dengue virus infection. J Neurol Sci 244: 117-122, 2006.

5. Soares CN, Faria LC, Peralta JM, de Freitas MR and Puccioni-Sohler M: Dengue infection: Neurological manifestations and cerebrospinal fluid (CSF) analysis. J Neurol Sci 249: 19-24, 2006.

6. Miranda de Sousa A, Puccioni-Sohler M, Dias Borges A, Fernandes Adorno L, Papais Alvarenga $M$ and Papais Alvarenga RM: Post-dengue neuromyelitis optica: Case report of a Japanese-descendent Brazilian child. J Infect Chemother 12: 396-398, 2006. 
7. Williams M: Manual muscle testing, development and current use. Phys Ther Rev 36: 797-805, 1956.

8. Seet RC, Lim EC and Wilder-Smith EP: Acute transverse myelitis following dengue virus infection. J Clin Virol 35: 310-312, 2006.

9. Larik A, Chiong Y, Lee LC and Ng YS: Longitudinally extensive transverse myelitis associated with dengue fever. BMJ Case Rep 2012: pii: bcr1220115378, 2012.

10. Chanthamat $\mathrm{N}$ and Sathirapanya P: Acute transverse myelitis associated with dengue viral infection. J Spinal Cord Med 33: 425-427, 2010.

11. Renganathan A, Ng WK and Tan CT: Transverse myelitis in association with dengue infection. Neurol J Southeast Asia 1: 61-63, 1996

12. Kunishige M, Mitsui T, Tan BH, Leong HN, Takasaki T, Kurane I, Mihara A and Matsumoto T: Preferential gray matter involvement in dengue myelitis. Neurology 63: 1980-1981, 2004.

13. Leão RN, Oikawa T, Rosa ES, Yamaki JT, Rodrigues SG, Vasconcelos HB, Sousa MRS, Tsukimata JK, Azevedo RSS and Vasconcelos PFC: Isolation of dengue 2 virus from a patient with central nervous system involvement (transverse myelitis). Rev Soc Bras Med Trop 35: 401-404, 2002.
14. Lee IK, Liu JW and Yang KD: Fatal dengue hemorrhagic fever in adults: Emphasizing the evolutionary pre-fatal clinical and laboratory manifestations. PLoS Negl Trop Dis 6: e1532, 2012.

15. Assir MZ, Jawa A and Ahmed HI: Expanded dengue syndrome: Subacute thyroiditis and intracerebral hemorrhage. BMC Infect Dis 12: 240, 2012.

16. Sindic CJ, Van Antwerpen MP and Goffette S: The intrathecal humoral immune response: Laboratory analysis and clinical relevance. Clin Chem Lab Med 39: 333-340, 2001.

17. Lazarus JH: Silent thyroiditis and subacute thyroiditis. In: Werner \& Ingbar's The Thyroid: A Fundamental and Clinical Text. Braverman LE and Utiger RD (eds). 7th edition. Lippincott-Raven, Philadelphia, PA,pp577-591, 1996.

18. Desailloud R and Hober D: Viruses and thyroiditis: An update. Virol J 6: 5, 2009.

19. Eylan E, Zmucky R and Sheba C: Mumps virus and subacute thyroiditis; Evidence of a causal association. Lancet 272: 1062-1063, 1957.

20. Felix-Davies D: Autoimmunisation in subacute thyroiditis. Lancet 1: 880-883, 1958. 